\title{
A Biogeography-Based Optimization Algorithm Hybridized with Tabu Search for the Quadratic Assignment Problem
}

\author{
Wee Loon Lim, ${ }^{1}$ Antoni Wibowo, ${ }^{2}$ Mohammad Ishak Desa, ${ }^{3}$ and Habibollah Haron ${ }^{1}$ \\ ${ }^{1}$ Faculty of Computing, Universiti Teknologi Malaysia (UTM), 81310 Johor Bahru, Johor, Malaysia \\ ${ }^{2}$ School of Quantitative Sciences, UUM College of Arts and Sciences, Universiti Utara Malaysia, 06010 Sintok, Kedah, Malaysia \\ ${ }^{3}$ Faculty of Information and Communication Technology, Universiti Teknikal Malaysia Melaka, Hang Tuah Jaya, \\ 76100 Durian Tunggal, Melaka, Malaysia
}

Correspondence should be addressed to Wee Loon Lim; weeloon87@gmail.com

Received 13 August 2015; Accepted 25 October 2015

Academic Editor: Ezequiel López-Rubio

Copyright ( 2016 Wee Loon Lim et al. This is an open access article distributed under the Creative Commons Attribution License, which permits unrestricted use, distribution, and reproduction in any medium, provided the original work is properly cited.

The quadratic assignment problem (QAP) is an NP-hard combinatorial optimization problem with a wide variety of applications. Biogeography-based optimization (BBO), a relatively new optimization technique based on the biogeography concept, uses the idea of migration strategy of species to derive algorithm for solving optimization problems. It has been shown that BBO provides performance on a par with other optimization methods. A classical BBO algorithm employs the mutation operator as its diversification strategy. However, this process will often ruin the quality of solutions in QAP. In this paper, we propose a hybrid technique to overcome the weakness of classical BBO algorithm to solve QAP, by replacing the mutation operator with a tabu search procedure. Our experiments using the benchmark instances from QAPLIB show that the proposed hybrid method is able to find good solutions for them within reasonable computational times. Out of 61 benchmark instances tested, the proposed method is able to obtain the best known solutions for 57 of them.

\section{Introduction}

The quadratic assignment problem (QAP) belongs to the class of combinatorial optimization. This problem concerns assigning a number of facilities to the same number of locations, with the objective to find a way of assignment such that the total cost involved is minimized. The total cost of the QAP is the product of flow and distances between the facilities.

Sahni and Gonzalez [1] have shown that QAP is NPhard. Therefore, unless $\mathrm{P}=\mathrm{NP}$, it is impossible to find optimal solutions in polynomial time. The complexity of QAP draws the interest of researchers worldwide over the past few decades. More than 300 papers have been published for the theory, applications, and solution techniques for the QAP [2]. Despite the extensive research done, QAP remains one of the most difficult combinatorial optimization problems. Generally, QAP instances with problem size $>30$ cannot be solved within reasonable computational times. In the literature, a lot of heuristic approaches to the QAP have been proposed. Some of the common methods are simulated annealing, tabu search (TS), genetic algorithm (GA), and ant colony optimization [3-10].

Among the exact methods for QAP, branch and bound (BB) algorithm is the most efficient $[11,12]$. Currently, the largest instance solved by BB algorithm is of size 36 [4]. Solving QAP instances of size 20 was impossible before 1990s. It was until 1994 when Mautor and Roucairol [13] gave the exact solutions for nug16 and els19. The BB algorithm of Brixius and Anstreicher [14] solved the nug25 instance after 13 days of CPU time using sequential processing. The kra30a was solved by Hahn and Krarup [15] after 99 days of work with a sequential workstation. Nystrom [16] provided the exact solutions for the ste36b and ste36c after 200 days of work using a distributed environment. The nug30 remained unsolved until 2002 when Anstreicher et al. [17] reported the exact solution with seven days of work using 650 processors. Basically, the exact methods require much 
more computational resources and time than the heuristic algorithms. This is the reason we did not use exact methods in this study.

The QAP has a wide variety in the real-world applications with facility layout problem being the most popular applied area. These include campus planning, backboard wiring, hospital planning, zoning in a forest, and the placement of electronic components. Besides, the QAP is also used for typewriter keyboard design, development of control boards, turbine runner problem, ranking of archaeology data, scheduling of production lines analysis of chemical reactions, data analysis, economic problems, VLSI circuit and system design, website structure, and optimizing of Arabic keyboard design. Comprehensive review of QAP can be referred to in $[2,18]$.

Biogeography is a study of the geographical distribution of biological organisms. The science of biogeography can be referred to in the work of two naturalists in the nineteenth century named Wallace [19] and Darwin [20]. In the 1960s, MacArthur and Wilson [21] started to work on mathematical models of biogeography. Their study focused on the distribution of species among neighboring habitats and how species migrate from one habitat to another. However, it was until 2008 when Simon [22] presented a new optimization method based on it, namely, biogeographybased optimization (BBO). In his work, Simon applied BBO to the problem of sensor selection for aircraft engine health estimation and the performance of $\mathrm{BBO}$ is at a par with other population-based methods such as ant colony optimization, differential evolution, GA, and particle swarm optimization (PSO). The good performance of $\mathrm{BBO}$ on the sensor selection problem proves that it can be successfully applied to practical problems.

In his next paper, Simon [23] showed that BBO outperforms GA when both of them have a low mutation rate. Furthermore, BBO has shown a good performance when being applied to real-world optimization problems such as classification of satellite images, groundwater detection, and the solving of complex economic load dispatch [24-26].

The good performance of $\mathrm{BBO}$ on complex optimization problems inspired us to apply this iterative and populationbased method to QAP. A classical BBO algorithm uses migration and mutation operators as its intensification and diversification strategy, respectively. During the iterated process of $\mathrm{BBO}$ algorithm, migration operator tends to improve every solution within the population while mutation operator increases the diversity among them. However, quite often the mutation operator will ruin the quality of solutions.

In order to overcome the weakness of mutation operator, we propose to replace it with a tabu search procedure. TS is a heuristic procedure for solving the optimization problems. A typical TS starts with defining a neighborhood, in which a given solution may move to and produce a new one. TS will evaluate each of the neighboring solutions and choose the move that improves the objective function the most. If there is no improving move, TS will choose one that weakens the objective function the least. The main difference of TS from other local search techniques is that it will use memory to store the recent moves. These moves are then forbidden and thus avoiding the search procedure to return to the local optimum just visited.

The characteristics of TS described above make it a suitable replacement for the mutation operator of classical BBO. Not only the diversity of the population is maintained but at the same time it prevents the quality of solutions within the population from being ruined. We choose to incorporate the robust tabu search (RTS) of Taillard [27] into our BBO algorithm for QAP because it is efficient and requires fewer parameters in its implementation.

In this paper, we propose a $\mathrm{BBO}$ algorithm hybridized with tabu search (BBOTS) for QAP. We apply it to the benchmark instances obtained from QAPLIB [28] with size ranging from 12 to 80 . The experimental results demonstrate that the proposed algorithm outperforms the classical $\mathrm{BBO}$ algorithm with mutation operator. Our hybrid algorithm is able to solve most of the benchmark instances of QAP tested.

\section{Problem Definition}

The QAP is an assignment model in which a set of facilities is supposed to be allocated to a set of locations, with each location having exactly one facility. The objective is to find a way of assignment such that the total cost involved is minimized. The total cost is calculated by multiplying the flow and the distances between facilities.

Let $n$ be the number of facilities and locations, given two $n \times n$ matrices as follows:

$$
\begin{aligned}
& F=\left(f_{i k}\right) \text {, where } f_{i k} \text { is the flow from facility } i \text { to facility } \\
& k ; \\
& D=\left(d_{j l}\right) \text {, where } d_{j l} \text { is the distance from location } j \text { to } \\
& \text { location } l \text {. }
\end{aligned}
$$

The Koopmans-Beckmann [29] form of the QAP can be formulated as follows:

$$
\min _{\varphi \in S_{n}}\left[\sum_{i=1}^{n} \sum_{k=1}^{n} f_{i k} d_{\varphi(i) \varphi(k)}\right],
$$

where $S_{n}$ is the set of all permutations of the integers $1,2, \ldots, n$. Each product of $f_{i k} d_{\varphi(i) \varphi(k)}$ is the cost for assigning facility $i$ to location $\varphi(i)$ and facility $k$ to location $\varphi(k)$. There are several ways in mathematics to represent an assignment. Throughout this paper, we use the representation of assignment by permutation.

\section{Methods}

In this section, we will first introduce $\mathrm{BBO}$ as general. This includes explaining the terminology used and its difference with another popular population-based optimization method, the GA. Next, we will explain the proposed algorithm in detail. We developed two BBO algorithms for QAP:

(i) a classical $\mathrm{BBO}$ algorithm with mutation operator,

(ii) a BBO algorithm hybridized with tabu search. 
Computational experiments were carried out to show the difference in performance between them. Note that the implementation of both algorithms is identical, except for the mutation step. Therefore, all the other steps of the algorithms are explained only once.

3.1. Biogeography-Based Optimization. BBO is a new algorithm inspired by biogeography, which studies the geographical distribution of biological organisms. MacArthur and Wilson [21] worked together on the mathematical models of biogeography in the 1960s. They focused primarily on the distribution of species among neighboring habitats and how species migrate from one habitat to another. Since then, biogeography has become a major area of research. However, it was until 2008 when Simon [22] generalized it to obtain a general-purpose optimization algorithm.

Just like other biology-based algorithms, for example, GA and PSO, BBO is a population-based algorithm in which a population of candidate solutions is used in the searching procedure for global optima. BBO has certain common features with another popular optimization algorithm, the GA. In GA, an individual within the population is called a chromosome and has its own fitness value. Likewise in $\mathrm{BBO}$, each individual is termed as a habitat and has its habitat suitability index (HSI) to evaluate its quality as a solution. Since we are dealing with a minimization problem, a low-HSI habitat represents a good solution and a high-HSI habitat is a poor solution instead. Each chromosome in GA consists of genes, while, for $\mathrm{BBO}$, each habitat is characterized by Suitability Index Variables (SIVs). There are two main operators in GA, which are crossover and mutation. Meanwhile, in $\mathrm{BBO}$, the main operators are migration and mutation. The migration operator consists of emigration and immigration. It is used to improve and evolve the habitats (solutions to the optimization problem) in the population. Solution features (SIVs) emigrate from low-HSI habitats (emigrating habitats) to high-HSI habitats (immigrating habitats). In other words, high-HSI habitats accept new features from low-HSI habitats through the immigration process. There are different alternatives for migration and mutation process of $\mathrm{BBO}$. The way we implement these two operators are explained in detail in the later part. Table 1 compares the characteristics of $\mathrm{BBO}$ and $\mathrm{GA}$.

The classical BBO algorithm proposed by Simon [22] can be described with the algorithm in the following.

\section{A Classical BBO Algorithm}

(1) Initialize the $\mathrm{BBO}$ parameters (this includes deriving a representation scheme for habitats, which is problems dependent, and also initializing the maximum migration rate, maximum mutation rate, and elitism parameter).

(2) Initialize a random set of habitats, corresponding to the potential solutions.

(3) Associate each habitat with immigration and emigration rate based on their HSI.
TABLE 1: Comparison of characteristics for BBO and GA.

\begin{tabular}{ll}
\hline BBO & GA \\
\hline Population-based & Population-based \\
Habitat & Chromosome \\
SIV & Gene \\
HSI & Fitness \\
Migration operator & Crossover operator \\
Mutation operator & Mutation operator \\
\hline
\end{tabular}

(4) Probabilistically perform migration to modify each one-elite habitat. Then, recompute each HSI.

(5) Associate each habitat with mutation rate based on their species count.

(6) Probabilistically perform mutation to modify each nonelite habitat. Then, recompute each HSI.

(7) Go to step (3) for the next iteration. Repeat until a predefined number of generations are reached, or after an acceptable solution is found.

\subsection{The Proposed Algorithm}

3.2.1. Representing Scheme of Habitats. As mention earlier, a habitat is represented by a permutation of integer $1,2, \ldots, n$, in which $n$ is the number of facilities and locations, that is, the problem size. For example, $(2,5,1,4,3)$ is a possible habitat for an instance of QAP with problem size of 5 . In this case, " 2 " of $(2,5,1,4,3)$ means facility 1 is placed at location 2 , and it is a SIV of this habitat. Likewise, " 5 " means facility 2 is placed at location 5 and so on.

3.2.2. Initialization of the $B B O$ Algorithm. The $\mathrm{BBO}$ algorithm starts with a population of randomly generated habitats. In our algorithm, a habitat is represented by a permutation of integers. A permutation is randomly generated and will only be inserted if it does not exist in the population yet. This is to avoid having duplicate habitats in the initial population and hence enhancing the diversity.

3.2.3. Selection Strategy for Migration. In $\mathrm{BBO}$, a good habitat (solution) is one with low HSI. Good habitats tend to share their features with poor habitat. This is done by migrating SIVs from emigrating habitats to immigrating habitats. In order to perform migration, we will first use immigration rates $\left(\lambda_{k}\right)$ of a habitat to decide whether to modify it, then we use emigration rates $\left(\mu_{k}\right)$ of other habitats to decide which of them should migrate a SIV to the first habitat.

According to the biogeography, the SIVs of a good habitat (with low HSI) tend to emigrate to a poor habitat (with high HSI). Therefore, a good habitat has relatively high $\mu_{k}$ and low $\lambda_{k}$, while a poor solution has relatively low $\mu_{k}$ and high $\lambda_{k}$. 
(1) for $i=1$ to $h$ do

(2) if rand $\in[0,1]<\lambda_{i}$ then

$\triangleright$ solution $H_{i}$ is selected

(3) for $j=1$ to $h$ do

(4) $\quad$ if rand $\in[0,1]<\mu_{j}$ then

(5) randomly select a SIV $\sigma$ from $H_{j}$

(6) replace a random SIV in $H_{i}$ with $\sigma$

$\triangleright$ solution $H_{j}$ is selected

Algorithm 1: Selection strategy of migration operator.

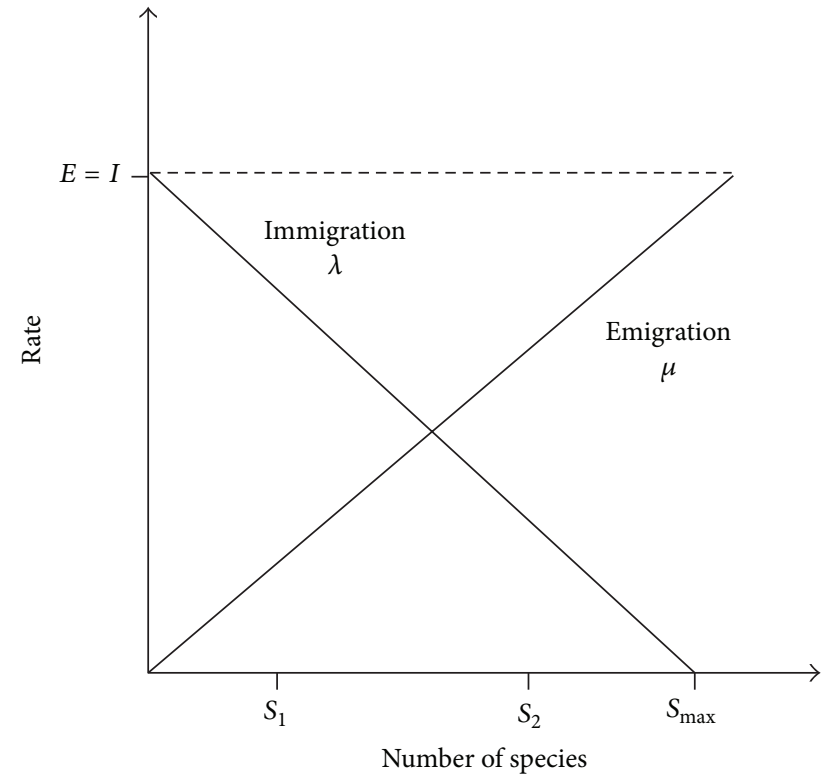

FIgURE 1: Model for immigration and emigration rates.

The immigration rate $\lambda_{k}$ and the emigration rates $\mu_{k}$ are calculated with the following equations, respectively,

$$
\begin{aligned}
& \lambda_{k}=I\left(1-\frac{k}{h}\right), \\
& \mu_{k}=\frac{E k}{h} .
\end{aligned}
$$

In the equations, $k$ represents the rank of a habitat after sorting them in accordance to their HSI. Habitats with high HSI (a poor solution) have lower rank while habitats with low HSI will have higher rank. In other words, the habitats are sorted from the worst to the best. In the equations, $h$ is the number of habitats in the population, while $I$ is the maximum immigration rate and $E$ the maximum emigration rate which are both usually set to 1 .

Figure 1 illustrates the model for immigration and emigration rates [22]. In the figure, $S_{1}$ is a relatively poor solution, while $S_{2}$ is a relatively good solution. Although in the figure the immigration and emigration rate are considered linear, they can actually be replaced with curves in which better performance might be attained. In fact, Mussetta and Pirinoli
[30] have shown that a variation of $\mathrm{BBO}$ with quadratic migration model and restart procedure outperforms the classical $\mathrm{BBO}$ when being applied to several benchmark functions. The selection strategy is summarized with the algorithm in Algorithm 1.

3.2.4. Selection Strategy for Mutation. In BBO, each habitat has an associated probability for them to exist as a solution to the given problem. The probability that whether mutation occurs in a habitat is called the mutation rate. To determine the mutation rate for each habitat, we must first evaluate the species count probability with the following equation:

$$
\mathbf{P}=\frac{\mathbf{v}}{\sum_{i=1}^{h+1} v_{i}},
$$

in which $v$ and $v_{i}$ are evaluated by

$$
\begin{aligned}
& \mathbf{v}=\left[\begin{array}{llll}
v_{1} & v_{2} & \cdots & v_{h+1}
\end{array}\right]^{T}, \\
& v_{i} \\
& = \begin{cases}\frac{h !}{(h+1-i) !(i-1) !} & \left(i=1,2, \ldots, i^{\prime}\right) \\
v_{h+2-i} & \left(i=i^{\prime}+1, i^{\prime}+2, \ldots, h+1\right),\end{cases}
\end{aligned}
$$

where $i^{\prime}=\operatorname{ceil}((n+1) / 2)$. The mutation rate $m(S)$ is inversely proportional to the species count probability; therefore, we have

$$
m(S)=m_{\max }\left(\frac{1-P_{s}}{P_{\max }}\right),
$$

where $m_{\max }$ is the maximum mutation rate and $P_{\max }$ the largest species count probability. The details on how to derive the above formulae can be referred to in [22].

3.2.5. Migration Operator. As mentioned earlier, the SIVs from a good habitat tend to migrate into a poor habitat. This migration operator is performed probabilistically based on immigration and emigration rates. In this section, we will explain how the migration is implemented in our BBOTS algorithm.

Consider dealing with an instance of QAP with problem size of 5. Suppose, based on immigration and emigration rates, that an emigrating habitat, $H_{e}=(2,4,3,5,1)$, and 


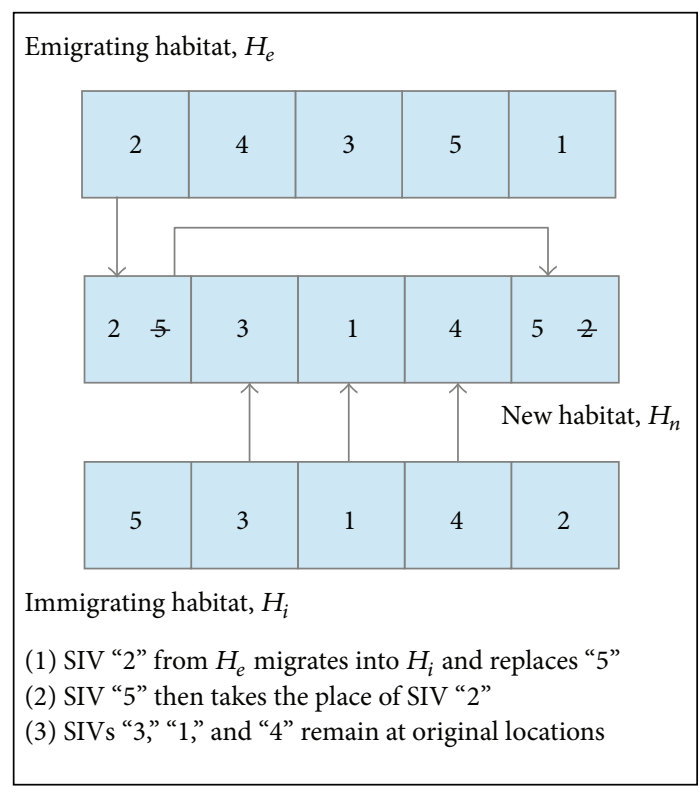

FIgURE 2: Migration operator of BBOTS algorithm.

an immigrating habitat, $H_{i}=(5,3,1,4,2)$, are selected. Therefore, a SIV of $H_{e}$ will be randomly selected and replaces a randomly selected SIV of $H_{i}$.

Assuming the first element of $H_{e}$, " 2 " is selected to replace the first SIV of $H_{i}$, "5." Therefore, the new habitat, $H_{n}=$ $(2,3,1,4,2)$, is produced. However, it is not a feasible solution for our problem. In order to maintain the feasibility of a solution, once a new SIV migrates into a habitat, the old SIV will replace the SIV with the same value of newly emigrating SIV. Therefore, the new habitat, $H_{n}$, will be $(2,3,1,4,5)$. Figure 2 illustrates this step clearly.

Once a new habitat is produced, it will only be accepted into the population only if it is not the same with any existing habitat. This is to enhance the diversity throughout the population. Besides, we use the concept of elitism to prevent the best solutions from being corrupted by immigration. This is done by setting the immigration rate for best solutions to 0 .

3.2.6. Mutation Operator. According to biogeography, cataclysmic events may happen from time to time, drastically changing the characteristics of a natural habitat. In $\mathrm{BBO}$ algorithm, this is imitated through a mutation operator. This process is important to increase the diversity among population.

In classical BBO algorithm, a mutation is performed by simply replacing a selected SIV of a habitat with a randomly generated SIV. Remember, in BBO, migration operator serves as intensification strategy, while mutation operator is used to maintain the diversity. Habitats within the population are improved by migration operator throughout the iteration of BBO algorithm. However, this effort might be ruined by the mutation operator because the quality of the mutated habitats is not guaranteed. Quite often, the mutation process will result in a poor habitat.

A simple solution for this drawback comes in mind such that after performing the mutation, the mutated habitats are kept in the population only if the quality is better than the original habitats. However, this is not practical when solving a complex optimization problem such as QAP. Most of the time, the resulting habitats from a simple mutation operator are unlikely to be better than the original habitats, especially as the algorithm converges.

To overcome this weakness of classical BBO algorithm, we propose to replace the mutation operator with a tabu search procedure. Proposed by Glover [31], TS is a metaheuristic which performs local search based on the information in the memory. TS is both neighborhood-based and iterative. At each iteration, current solution will make a move to the neighborhood solution with the best objective function value. To avoid trapping in local optima, the move that has been made will be stored in a tabu list and a reverse move to previous solutions is forbidden. The performance of tabu search highly depends on the neighborhood type used and tabu list implementation.

The advantages of replacing the mutation operator of classical BBO algorithm with TS come in two points. First, the original aim of mutation process is maintained, which is to increase the diversity of the population. Besides, at the same time, the quality of the resulting habitats is prevented from being ruined.

In order to prove the above statement, we developed two $\mathrm{BBO}$ algorithms for QAP:

(i) a classical $\mathrm{BBO}$ algorithm with mutation operator,

(ii) a $\mathrm{BBO}$ algorithm hybridized with tabu search.

For the classical BBO algorithm, the SIV of a habitat will be chosen to undergo mutation based on the mutation rate. The mutation process is then performed by replacing the old SIV with another randomly generated SIV. After that, SIV of the habitat, which is of the same value with the randomly generated SIV, will inherit the original value of the mutated SIV. Thus, the resulting habitat will remain a feasible solution. Figure 3 illustrates the mutation process of the classical BBO algorithm for QAP.

For the proposed BBOTS algorithm, we chose to replace the mutation operator with robust tabu search (RTS) of Taillard [27]. Despite being developed long time ago (1991), RTS is still one of the best performing algorithms for QAP.

The tabu list of RTS consists of pairs of facilities that cannot be exchanged. In the tabu list, the latest iteration at which a pair of facilities is placed at certain locations is stored. A swap of a pair of facilities is taboo for a number of iterations if they were swapped in the last iteration. However, a taboo move will be allowed if the new solution has a better objective function value than the current best solution. The tabu tenure 


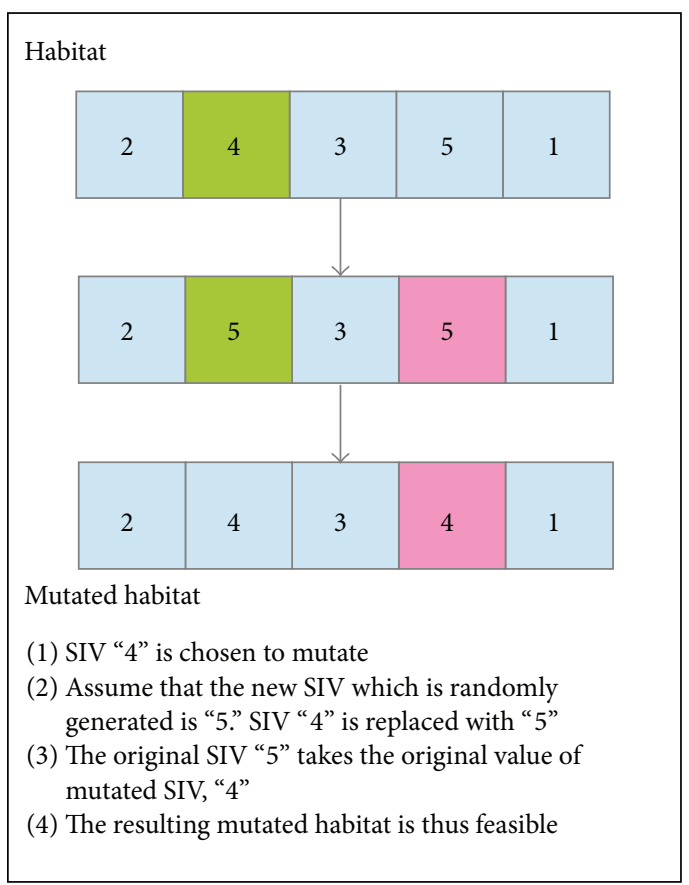

FIgURE 3: The mutation process of classical BBO algorithm for QAP.

of RTS changes between $0.9 n$ and $1.1 n$ dynamically during the procedure.

The advantages of RTS as compared to other adaptions of tabu search for QAP are that RTS is both efficient and robust. The robustness of RTS means that it requires less complexity and fewer parameters in its implementation. Therefore as we incorporate RTS into our BBOTS algorithm, we do not have to alter the parameter values for every benchmark instance of QAP tested.

In BBOTS algorithm, the mutation rate is now the probability that a habitat in the population will undergo RTS procedure. The number of iterations of RTS is set to $n$, which is the size of a problem. As compared to the original RTS, the number of iterations we use is relatively much lower. This is because the use of RTS in our BBOTS algorithm is limited to a quick search only. The resulting habitat after the RTS procedure will replace the original habitat if and only if the population does not contain a same habitat yet.

3.2.7. BBOTS Algorithm for QAP. The BBOTS algorithm for QAP is shown in Algorithm 2.

\section{Results and Discussion}

Because of the nature of heuristic algorithms, in order to evaluate the performance of a new algorithm, it is a common practice to test it with the benchmark instances of the problem and compare its results with other existing methods. For QAP, there are benchmark instances of various sizes available from the QAPLIB. These instances are used in most
TABLE 2: Parameter setting of classical BBO and BBOTS algorithms for QAP.

\begin{tabular}{ll}
\hline Parameter & Value \\
\hline Population size & 100 \\
Number of iterations & 300 \\
Maximum immigration rate & 1 \\
Maximum emigration rate & 1 \\
Maximum mutation rate & 0.1 \\
Number of elites & 2 \\
\hline
\end{tabular}

of the literature. The benchmark instances of QAP can be classified into four types:

(i) Type I: real-life instances obtained from practical applications of QAP,

(ii) Type II: unstructured, randomly generated instances for which the distance and flow matrices are randomly generated based on a uniform distribution,

(iii) Type III: randomly generated instances with structure that is similar to that of real-life instances,

(iv) Type IV: instances in which distances are based on the Manhattan distance on a grid.

The computational results are reported through 3 stages. First, we compare the results of classical $\mathrm{BBO}$ algorithm with mutation operator and the proposed BBOTS by applying them to selected benchmark instances from QAPLIB. After that, we further the experiments of BBOTS algorithm by testing with even more benchmark instances of QAP. Lastly, we compare the BBOTS algorithm with other state-of-the-art methods.

Both the classical $\mathrm{BBO}$ algorithm with mutation operator and BBOTS for QAP are programmed in MATLAB, running on the same machine with an Intel Core i3-2120 processor at $3.3 \mathrm{GHz}$. In order to fairly compare their performance, the parameter setting for both of the algorithms is the same throughout all the instances tested. The values of parameters used are indicated in Table 2.

We compare the performance of classical $\mathrm{BBO}$ and BBOTS algorithm for QAP by applying them on 37 benchmark instances. The computational results are shown in Table 3. The performance of the algorithms is evaluated with the following criteria:

(i) the best solution found over 10 runs,

(ii) the average deviation from the optimal or best known solution, $\bar{\delta}=100(\bar{z}-z) / z(\%)$, where $\bar{z}$ is the average objective function value and $z$ is the best known solution value, 


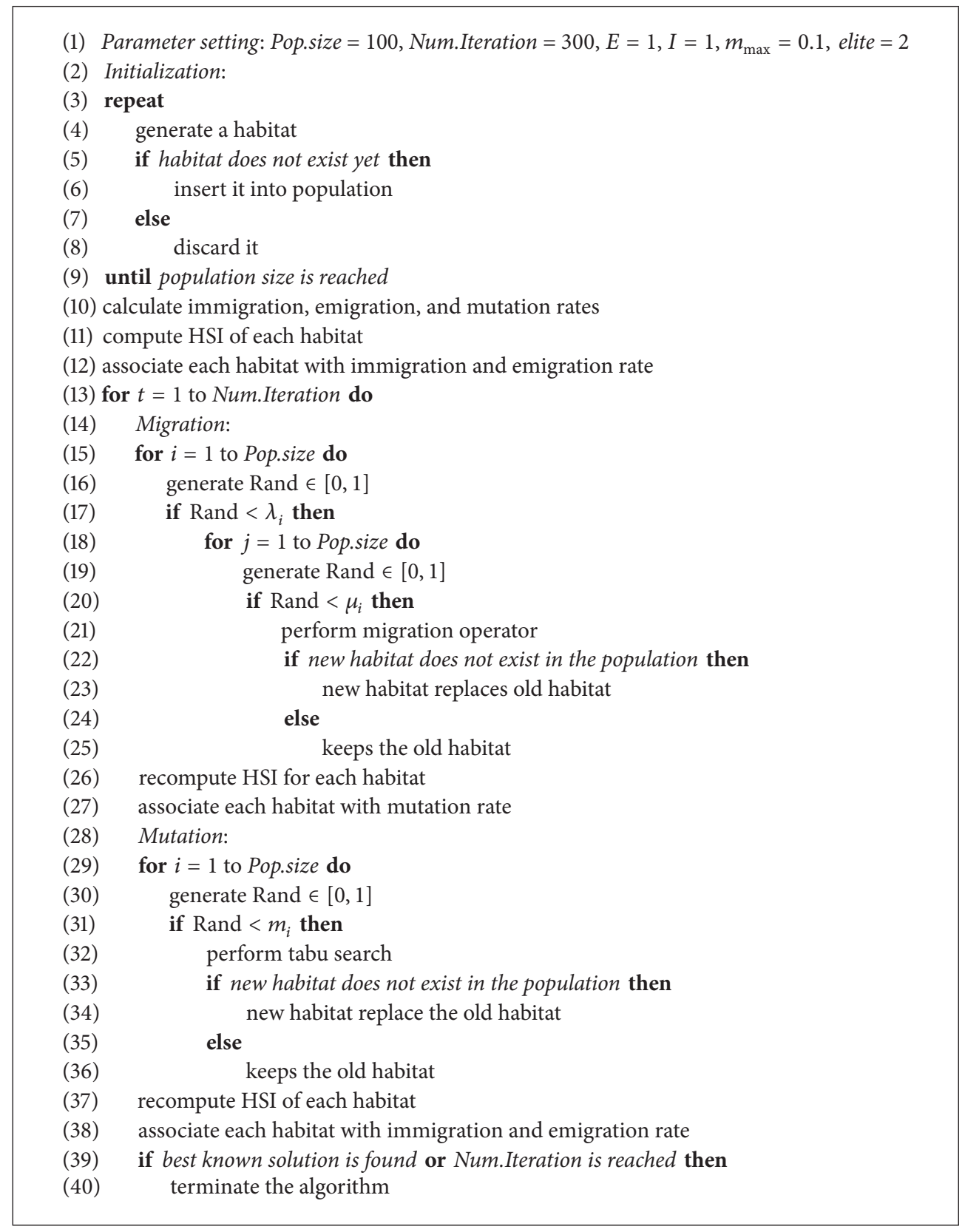

Algorithm 2: BBOTS algorithm for QAP.

(iii) the number of times (\#) in which the optimal or best known solution is reached.

The benchmark instances used are of different types. Regardless of the types of instances tested, BBOTS algorithm clearly performs much better than the classical $\mathrm{BBO}$ with mutation operator. BBOTS algorithm is able to find the optimal or best known solutions for 36 instances out of a total of 37 , while the classical BBO algorithm only manages to get 2. The average deviations from the best known solutions of BBOTS are also much lower for all the benchmark instances tested.
We further the experiments of our BBOTS algorithm by applying it to more benchmark instances of QAP. Table 4 shows the results obtained by BBOTS algorithm for 61 benchmark instances with size ranging from 12 to 80 . The parameter values used are the same as those used previously. The BBOTS algorithm is terminated at 300 iterations or when the best known solutions are found, whichever comes first.

Computational results show that the proposed BBOTS algorithm is able to find optimal or best known solutions for 57 QAP benchmark instances out of a total of 61. Most of the times, BBOTS is able to solve the instances tested on every single run. The average deviations from the best known 
TABLE 3: Comparative results between classical BBO and BBOTS algorithms for QAP.

\begin{tabular}{|c|c|c|c|c|c|c|c|}
\hline \multirow{2}{*}{ Instance } & \multirow{2}{*}{ Best known solution } & \multicolumn{3}{|c|}{$\mathrm{BBO}$} & \multicolumn{3}{|c|}{ BBOTS } \\
\hline & & Best solution & $\bar{\delta}$ & $\#$ & Best solution & $\bar{\delta}$ & \# \\
\hline chr12a & 9552 & 9988 & 33.654 & 0 & 9552 & 0.000 & 10 \\
\hline chr12b & 9742 & 9942 & 28.337 & 0 & 9742 & 0.000 & 10 \\
\hline chr12c & 11156 & 12336 & 32.854 & 0 & 11156 & 0.000 & 10 \\
\hline chr15a & 9896 & 14496 & 97.409 & 0 & 9896 & 0.000 & 10 \\
\hline chr15b & 7990 & 15298 & 136.683 & 0 & 7990 & 0.298 & 9 \\
\hline chr15c & 9504 & 18392 & 112.822 & 0 & 9504 & 0.000 & 10 \\
\hline chr18a & 11098 & 29870 & 190.483 & 0 & 11098 & 0.079 & 8 \\
\hline chr18b & 1534 & 2170 & 51.356 & 0 & 1534 & 0.000 & 10 \\
\hline els19 & 17212548 & 21315378 & 34.293 & 0 & 17212548 & 0.000 & 10 \\
\hline escl6a & 68 & 70 & 8.235 & 0 & 68 & 0.000 & 10 \\
\hline escl6b & 292 & 292 & 0.000 & 10 & 292 & 0.000 & 10 \\
\hline esc16c & 160 & 164 & 5.875 & 0 & 160 & 0.000 & 10 \\
\hline esc16d & 16 & 18 & 22.500 & 0 & 16 & 0.000 & 10 \\
\hline had12 & 1652 & 1662 & 1.877 & 0 & 1652 & 0.000 & 10 \\
\hline had14 & 2724 & 2762 & 2.349 & 0 & 2724 & 0.000 & 10 \\
\hline had16 & 3720 & 3820 & 3.468 & 0 & 3720 & 0.000 & 10 \\
\hline had18 & 5358 & 5500 & 3.763 & 0 & 5358 & 0.000 & 10 \\
\hline had20 & 6922 & 7156 & 4.143 & 0 & 6922 & 0.000 & 10 \\
\hline nug12 & 578 & 590 & 6.574 & 0 & 578 & 0.000 & 10 \\
\hline nug14 & 1014 & 1086 & 9.310 & 0 & 1014 & 0.000 & 10 \\
\hline nug15 & 1150 & 1250 & 11.078 & 0 & 1150 & 0.000 & 10 \\
\hline nug16a & 1610 & 1762 & 11.503 & 0 & 1610 & 0.000 & 10 \\
\hline nug16b & 1240 & 1374 & 12.694 & 0 & 1240 & 0.000 & 10 \\
\hline nug17 & 1732 & 1914 & 12.125 & 0 & 1732 & 0.012 & 9 \\
\hline nug18 & 1930 & 2130 & 12.114 & 0 & 1930 & 0.000 & 10 \\
\hline nug20 & 2570 & 2868 & 13.416 & 0 & 2570 & 0.000 & 10 \\
\hline roul2 & 235528 & 247850 & 7.057 & 0 & 235528 & 0.000 & 10 \\
\hline rou15 & 354210 & 389802 & 12.105 & 0 & 354210 & 0.000 & 10 \\
\hline rou 20 & 725522 & 810284 & 12.490 & 0 & 725522 & 0.062 & 4 \\
\hline scr12 & 31410 & 31410 & 8.243 & 1 & 31410 & 0.000 & 10 \\
\hline scr15 & 51140 & 58958 & 22.004 & 0 & 51140 & 0.000 & 10 \\
\hline scr20 & 110030 & 146230 & 37.559 & 0 & 110030 & 0.000 & 10 \\
\hline tai10a & 135028 & 137362 & 2.974 & 0 & 135028 & 0.000 & 10 \\
\hline tail2a & 224416 & 230704 & 8.210 & 0 & 224416 & 0.000 & 10 \\
\hline tail5a & 388214 & 404108 & 8.843 & 0 & 388214 & 0.000 & 10 \\
\hline tail7a & 491812 & 540308 & 11.651 & 0 & 491812 & 0.093 & 8 \\
\hline tai20a & 703482 & 789348 & 13.658 & 0 & 705622 & 0.677 & 0 \\
\hline
\end{tabular}

solutions over 10 runs are at most $2.788 \%$. Even on large instance like tai80a, the average deviation is less than $3 \%$.

For indicative purpose, we compare the results of our BBOTS algorithm with 4 state-of-the-art QAP approaches in the literature:

(i) Iterated Tabu Search (ITS) algorithm by Misevicius (2012) [6]: the reported results are obtained using an Intel Pentium $900 \mathrm{MHz}$ single-core processor; (ii) hybrid metaheuristics combining greedy randomized adaptive search procedure and simulated annealing and tabu search (SA-TS) by Gunawan et al. (2014) [32]: the reported results are obtained using a 2.67 GHz Intel Core 2 Duo CPU;

(iii) information combination based evolutionary algorithm (ICEA) by Sun et al. (2014) [33]; 
TABLE 4: Computational results of BBOTS algorithm on benchmark instances of QAP.

\begin{tabular}{|c|c|c|c|c|}
\hline Instance & Best known solution & Best solution & $\bar{\delta}$ & $\#$ \\
\hline bur26a & 5426670 & 5426670 & 0.028 & 5 \\
\hline bur26b & 3817852 & 3817852 & 0.000 & 10 \\
\hline bur26c & 5426795 & 5426795 & 0.000 & 10 \\
\hline bur26d & 3821225 & 3821225 & 0.000 & 10 \\
\hline bur26e & 5386879 & 5386879 & 0.000 & 10 \\
\hline bur26f & 3782044 & 3782044 & 0.000 & 10 \\
\hline bur26g & 10117172 & 10117172 & 0.000 & 10 \\
\hline bur26h & 7098658 & 7098658 & 0.000 & 10 \\
\hline $\operatorname{chr} 12 \mathrm{a}$ & 9552 & 9552 & 0.000 & 10 \\
\hline chr12b & 9742 & 9742 & 0.000 & 10 \\
\hline $\operatorname{chr} 12 \mathrm{c}$ & 11156 & 11156 & 0.000 & 10 \\
\hline chr15a & 9896 & 9896 & 0.000 & 10 \\
\hline chr15b & 7990 & 7990 & 0.298 & 9 \\
\hline $\operatorname{chr} 15 \mathrm{c}$ & 9504 & 9504 & 0.000 & 10 \\
\hline chr18a & 11098 & 11098 & 0.079 & 8 \\
\hline chr18b & 1534 & 1534 & 0.000 & 10 \\
\hline chr20a & 2192 & 2192 & 0.876 & 3 \\
\hline chr20c & 14142 & 14142 & 0.604 & 9 \\
\hline els19 & 17212548 & 17212548 & 0.000 & 10 \\
\hline esc16a & 68 & 68 & 0.000 & 10 \\
\hline esc16b & 292 & 292 & 0.000 & 10 \\
\hline esc16c & 160 & 160 & 0.000 & 10 \\
\hline esc16d & 16 & 16 & 0.000 & 10 \\
\hline had12 & 1652 & 1652 & 0.000 & 10 \\
\hline had14 & 2724 & 2724 & 0.000 & 10 \\
\hline had16 & 3720 & 3720 & 0.000 & 10 \\
\hline had18 & 5358 & 5358 & 0.000 & 10 \\
\hline had20 & 6922 & 6922 & 0.000 & 10 \\
\hline kra30a & 88900 & 88900 & 0.090 & 9 \\
\hline kra30b & 91420 & 91420 & 0.060 & 6 \\
\hline kra32 & 88700 & 88700 & 0.311 & 7 \\
\hline nug12 & 578 & 578 & 0.000 & 10 \\
\hline nug14 & 1014 & 1014 & 0.000 & 10 \\
\hline nug15 & 1150 & 1150 & 0.000 & 10 \\
\hline nug16a & 1610 & 1610 & 0.000 & 10 \\
\hline nug16b & 1240 & 1240 & 0.000 & 10 \\
\hline nug17 & 1732 & 1732 & 0.012 & 9 \\
\hline nug18 & 1930 & 1930 & 0.000 & 10 \\
\hline nug20 & 2570 & 2570 & 0.000 & 10 \\
\hline nug21 & 2438 & 2438 & 0.000 & 10 \\
\hline nug22 & 3596 & 3596 & 0.000 & 10 \\
\hline nug24 & 3488 & 3488 & 0.000 & 10 \\
\hline nug25 & 3744 & 3744 & 0.000 & 10 \\
\hline nug27 & 5234 & 5234 & 0.000 & 10 \\
\hline nug28 & 5166 & 5166 & 0.209 & 4 \\
\hline nug30 & 6124 & 6124 & 0.065 & 2 \\
\hline rou12 & 235528 & 235528 & 0.000 & 10 \\
\hline
\end{tabular}

TABle 4: Continued.

\begin{tabular}{lcccc}
\hline Instance & Best known solution & Best solution & $\bar{\delta}$ & $\#$ \\
\hline rou15 & 354210 & 354210 & 0.000 & 10 \\
rou20 & 725522 & 725522 & 0.062 & 4 \\
scr12 & 31410 & 31410 & 0.000 & 10 \\
scr15 & 51140 & 51140 & 0.000 & 10 \\
scr20 & 110030 & 110030 & 0.000 & 10 \\
sko42 & 15812 & 15812 & 0.028 & 9 \\
tai10a & 135028 & 135028 & 0.000 & 10 \\
tai12a & 224416 & 224416 & 0.000 & 10 \\
tai15a & 388214 & 388214 & 0.000 & 10 \\
tail7a & 491812 & 491812 & 0.093 & 8 \\
tai20a & 703482 & 705622 & 0.677 & 0 \\
tai30a & 1818146 & 1843224 & 1.795 & 0 \\
tai80a & 13499184 & 13841214 & 2.788 & 0 \\
wil50 & 48816 & 48848 & 0.117 & 0 \\
\hline
\end{tabular}

(iv) genetic algorithm with a new recombination operator (GAR) by Tosun (2014) [8]: the reported results are obtained using a $2.21 \mathrm{GHz}$ AMD Athlon (TM) $64 \times 2$ dual processor.

Since there are differences in computing hardware, termination criterion, and result reporting method, comparing the results of each algorithm is not a straightforward task. Therefore, the comparison in Table 5 should be interpreted cautiously.

The average deviations from the optimal or best known solutions are over 10 runs except for SA-TS and ICEA, which were executed for 20 and 30 times, respectively. The number of times in which the optimal or best known solutions are reached is indicated in the parentheses, if known. Should the particular authors not report their results on certain benchmark instances, the cells are left empty.

\section{Conclusion}

In this paper, we presented a biogeography-based optimization algorithm hybridized with tabu search for QAP. A classical BBO algorithm uses the mutation operator as its diversification strategy. This step often destroys the quality of habitats within the population. In the proposed BBOTS algorithm, we replaced the mutation operator with robust tabu search. The diversity among the population is maintained and, at the same time, the quality of habitats is prevented from being ruined.

The comparative results showed that BBOTS algorithm outperforms the classical BBO with mutation operator when tested with benchmark instances of QAP. The proposed BBOTS algorithm is able to obtain the optimal or best known solutions for many of the benchmark instances drawn from the QAPLIB. In particular, out of a total of 61 benchmark instances evaluated, BBOTS is able to reach the current best known solutions for 57 of them. 
TABLE 5: Comparative results between BBOTS algorithm and state-of-the-art QAP approaches.

\begin{tabular}{|c|c|c|c|c|c|c|}
\hline Instance & Best known solution & BBOTS & ITS & SA-TS & ICEA & GAR \\
\hline bur26a & 5426670 & $0.028(5)$ & & & 0.000 & 0.92 \\
\hline bur26b & 3817852 & $0.000(10)$ & & & & 0.65 \\
\hline bur26c & 5426795 & $0.000(10)$ & & & & 1.31 \\
\hline bur26d & 3821225 & $0.000(10)$ & & & & 0.56 \\
\hline bur26e & 5386879 & $0.000(10)$ & & & & 1.08 \\
\hline bur26f & 3782044 & $0.000(10)$ & & & & 0.56 \\
\hline bur26g & 10117172 & $0.000(10)$ & & & & 0.74 \\
\hline bur26h & 7098658 & $0.000(10)$ & & & & \\
\hline $\operatorname{chr} 12 \mathrm{a}$ & 9552 & $0.000(10)$ & & 0.00 & & \\
\hline chr12b & 9742 & $0.000(10)$ & & 0.00 & & \\
\hline $\operatorname{chr} 12 \mathrm{c}$ & 11156 & $0.000(10)$ & & 0.00 & & \\
\hline $\operatorname{chr} 15 \mathrm{a}$ & 9896 & $0.000(10)$ & & 0.00 & & \\
\hline chr15b & 7990 & $0.298(9)$ & & 0.00 & & \\
\hline $\operatorname{chr} 15 \mathrm{c}$ & 9504 & $0.000(10)$ & & 0.00 & & \\
\hline chr18a & 11098 & $0.079(8)$ & & 0.00 & & \\
\hline chr18b & 1534 & $0.000(10)$ & & 0.00 & & \\
\hline chr20a & 2192 & $0.876(3)$ & & 1.50 & & \\
\hline chr20c & 14142 & $0.604(9)$ & & 0.00 & & \\
\hline els19 & 17212548 & $0.000(10)$ & $0.00(10)$ & & & \\
\hline esc16a & 68 & $0.000(10)$ & & & & 0.00 \\
\hline esc16b & 292 & $0.000(10)$ & & & & 0.00 \\
\hline esc16c & 160 & $0.000(10)$ & & & & 0.00 \\
\hline esc16d & 16 & $0.000(10)$ & & & & 0.00 \\
\hline had12 & 1652 & $0.000(10)$ & & 0.00 & & 0.00 \\
\hline had14 & 2724 & $0.000(10)$ & & 0.40 & & 0.07 \\
\hline had16 & 3720 & $0.000(10)$ & & 0.03 & & 0.38 \\
\hline had18 & 5358 & $0.000(10)$ & & 0.00 & & 0.56 \\
\hline had20 & 6922 & $0.000(10)$ & & 0.08 & & 1.39 \\
\hline kra30a & 88900 & $0.090(9)$ & $0.01(8)$ & 0.74 & 0.000 & \\
\hline kra30b & 91420 & $0.060(6)$ & $0.00(10)$ & 0.00 & 0.000 & \\
\hline kra32 & 88700 & $0.311(7)$ & & 0.00 & & \\
\hline nug12 & 578 & $0.000(10)$ & & 0.00 & & \\
\hline nug14 & 1014 & $0.000(10)$ & & 0.00 & & \\
\hline nug15 & 1150 & $0.000(10)$ & & 0.00 & & \\
\hline nug16a & 1610 & $0.000(10)$ & & & & \\
\hline nug16b & 1240 & $0.000(10)$ & & & & \\
\hline nug17 & 1732 & $0.012(9)$ & & & & \\
\hline nug18 & 1930 & $0.000(10)$ & & & & 4.97 \\
\hline nug20 & 2570 & $0.000(10)$ & & 0.00 & & \\
\hline nug21 & 2438 & $0.000(10)$ & & 0.00 & & \\
\hline nug22 & 3596 & $0.000(10)$ & & 0.00 & & \\
\hline nug24 & 3488 & $0.000(10)$ & & 0.00 & & \\
\hline nug25 & 3744 & $0.000(10)$ & & 0.00 & & \\
\hline nug27 & 5234 & $0.000(10)$ & & 0.00 & & \\
\hline nug28 & 5166 & $0.209(4)$ & & 0.02 & & \\
\hline nug30 & 6124 & $0.065(2)$ & $0.00(10)$ & 0.01 & 0.000 & \\
\hline rou12 & 235528 & $0.000(10)$ & & 0.00 & & \\
\hline rou15 & 354210 & $0.000(10)$ & & 0.00 & & \\
\hline rou 20 & 725522 & $0.062(4)$ & & 0.03 & & \\
\hline scr12 & 31410 & $0.000(10)$ & & 0.00 & & \\
\hline
\end{tabular}


TABLE 5: Continued.

\begin{tabular}{|c|c|c|c|c|c|c|}
\hline Instance & Best known solution & BBOTS & ITS & SA-TS & ICEA & GAR \\
\hline scr15 & 51140 & $0.000(10)$ & & 0.00 & & \\
\hline scr20 & 110030 & $0.000(10)$ & & 0.00 & & \\
\hline sko42 & 15812 & $0.028(9)$ & $0.00(10)$ & 0.14 & 0.000 & \\
\hline tail0a & 135028 & $0.000(10)$ & & 0.00 & & \\
\hline tail2a & 224416 & $0.000(10)$ & & 0.00 & & \\
\hline tail5a & 388214 & $0.000(10)$ & & 0.00 & & \\
\hline tail7a & 491812 & $0.093(8)$ & & 0.00 & & \\
\hline tai20a & 703482 & $0.677(0)$ & $0.00(10)$ & 0.16 & 0.168 & \\
\hline tai30a & 1818146 & $1.795(0)$ & $0.00(10)$ & 1.51 & 0.276 & \\
\hline tai80a & 13499184 & $2.788(0)$ & $0.36(1)$ & 3.57 & 0.998 & 10.94 \\
\hline wil50 & 48816 & $0.117(0)$ & $0.06(4)$ & 0.11 & & 7.18 \\
\hline
\end{tabular}

\section{Conflict of Interests}

The authors declare that there is no conflict of interests regarding the publication of this paper.

\section{Acknowledgment}

This research is funded by Universiti Teknologi Malaysia under Fundamental Research Grant Scheme (Vote Project no. R.J130000.7828.4F497).

\section{References}

[1] S. Sahni and T. Gonzalez, "P-complete approximation problems," Journal of the Association for Computing Machinery, vol. 23, no. 3, pp. 555-565, 1976.

[2] E. M. Loiola, N. M. M. de Abreu, P. O. Boaventura-Netto, P. Hahn, and T. Querido, "A survey for the quadratic assignment problem," European Journal of Operational Research, vol. 176, no. 2, pp. 657-690, 2007.

[3] J.-C. Wang, "A multistart simulated annealing algorithm for the quadratic assignment problem," in Proceedings of the $3 \mathrm{rd}$ IEEE International Conference on Innovations in Bio-Inspired Computing and Applications (IBICA '12), pp. 19-23, Kaohsiung, Taiwan, September 2012.

[4] U. Tosun, "On the performance of parallel hybrid algorithms for the solution of the quadratic assignment problem," Engineering Applications of Artificial Intelligence, vol. 39, pp. 267-278, 2015.

[5] M. Czapiński, "An effective parallel multistart tabu search for quadratic assignment problem on CUDA platform," Journal of Parallel and Distributed Computing, vol. 73, no. 11, pp. 1461-1468, 2013.

[6] A. Misevicius, "An implementation of the iterated tabu search algorithm for the quadratic assignment problem," OR Spectrum, vol. 34, no. 3, pp. 665-690, 2012.

[7] U. Tosun, T. Dokeroglu, and A. Cosar, "A robust island parallel genetic algorithm for the quadratic assignment problem," International Journal of Production Research, vol. 51, no. 14, pp. 41174133, 2013.

[8] U. Tosun, "A new recombination operator for the genetic algorithm solution of the quadratic assignment problem," Procedia Computer Science, vol. 32, pp. 29-36, 2014.

[9] M. Lstibůrek, J. Stejskal, A. Misevicius, J. Korecký, and Y. A. ElKassaby, "Expansion of the minimum-inbreeding seed orchard design to operational scale," Tree Genetics and Genomes, vol. 11, no. 1, pp. 1-8, 2015.

[10] A. S. Ramkumar, S. G. Ponnambalam, and N. Jawahar, "A population-based hybrid ant system for quadratic assignment formulations in facility layout design," International Journal of Advanced Manufacturing Technology, vol. 44, no. 5-6, pp. 548558, 2009.

[11] R. E. Burkard, E. Cela, P. M. Pardalos, and L. Pitsoulis, "The quadratic assignment problem," in Handbook of Combinatorial Optimization, P. M. Pardalos and D. Z. Du, Eds., pp. 241-338, Kluwer Academic Publishers, 1998.

[12] P. C. Gilmore, "Optimal and suboptimal algorithms for the quadratic assignment problem," Journal of the Society for Industrial and Applied Mathematics, vol. 10, no. 2, pp. 305-313, 1962.

[13] T. Mautor and C. Roucairol, "A new exact algorithm for the solution of quadratic assignment problems," Discrete Applied Mathematics, vol. 55, no. 3, pp. 281-293, 1994.

[14] N. W. Brixius and K. M. Anstreicher, "Solving quadratic assignment problems using convex quadratic programming relaxations," Optimization Methods and Software, vol. 16, no. 14, pp. 49-68, 2001.

[15] P. M. Hahn and J. Krarup, "A hospital facility layout problem finally solved," Journal of Intelligent Manufacturing, vol. 12, no. 5, pp. 487-496, 2001.

[16] M. Nystrom, Solving Certain Large Instances of the Quadratic Assignment Problem: Steinberg's Examples, California Institute of Technology, Pasadena, Calif, USA, 1999.

[17] K. Anstreicher, N. Brixius, J.-P. Goux, and J. Linderoth, "Solving large quadratic assignment problems on computational grids," Mathematical Programming, vol. 91, no. 3, pp. 563-588, 2002.

[18] Z. Drezner, P. M. Hahn, and É. D. Taillard, "Recent advances for the quadratic assignment problem with special emphasis on instances that are difficult for meta-heuristic methods," Annals of Operations Research, vol. 139, pp. 65-94, 2005.

[19] A. Wallace, The Geographical Distribution of Animals (Two Volumes), Adamant Media Corporation, Boston, Mass, USA, 2005.

[20] C. Darwin, On the Origin of Species, Gramercy, New York, NY, USA, 1995.

[21] R. MacArthur and E. Wilson, The Theory of Biogeography, Princeton University Press, Princeton, NJ, USA, 1967.

[22] D. Simon, "Biogeography-based optimization," IEEE Transactions on Evolutionary Computation, vol. 12, no. 6, pp. 702-713, 2008. 
[23] D. Simon, "A probabilistic analysis of a simplified biogeographybased optimization algorithm," Evolutionary Computation, vol. 19, no. 2, pp. 167-188, 2011.

[24] V. Panchal, P. Singh, N. Kaur, and H. Kundra, "Biogeography based satellite image classification," International Journal of Information Security, vol. 6, no. 2, pp. 269-274, 2009.

[25] V. Panchal, H. Kundra, and A. Kaur, "An integrated approach to biogeography based optimization with case based reasoning for retrieving groundwater possibility," International Journal of Computer Applications, vol. 1, no. 8, pp. 975-8887, 2010.

[26] A. Bhattacharya and P. K. Chattopadhyay, "Solving complex economic load dispatch problems using biogeography-based optimization," Expert Systems with Applications, vol. 37, no. 5, pp. 3605-3615, 2010.

[27] E. Taillard, "Robust taboo search for the quadratic assignment problem," Parallel Computing. Theory and Applications, vol. 17, no. 4-5, pp. 443-455, 1991.

[28] R. E. Burkard, E. Cela, S. E. Karisch, and F. Rendl, QAPLIB Home Page, 2004, http://anjos.mgi.polymtl.ca/qaplib/.

[29] T. C. Koopmans and M. Beckmann, "Assignment problems and the location of economic activities," Econometrica, vol. 25, no. 1, pp. 53-76, 1957.

[30] M. Mussetta and P. Pirinoli, "MmCn-BBO schemes for electromagnetic problem optimization," in Proceedings of the 7th European Conference on Antennas and Propagation (EuCAP '13), pp. 1058-1059, Gothenburg, Sweden, April 2013.

[31] F. Glover, "Future paths for integer programming and links to artificial intelligence," Computers \& Operations Research, vol. 13, no. 5, pp. 533-549, 1986.

[32] A. Gunawan, K. M. Ng, K. L. Poh, and H. C. Lau, "Hybrid metaheuristics for solving the quadratic assignment problem and the generalized quadratic assignment problem," in Proceedings of the IEEE International Conference on Automation Science and Engineering (CASE '14), pp. 119-124, Taipei, Taiwan, August 2014.

[33] J. Sun, Q. Zhang, and X. Yao, "Meta-heuristic combining prior online and offline information for the quadratic assignment problem," IEEE Transactions on Cybernetics, vol. 44, no. 3, pp. 429-444, 2014. 

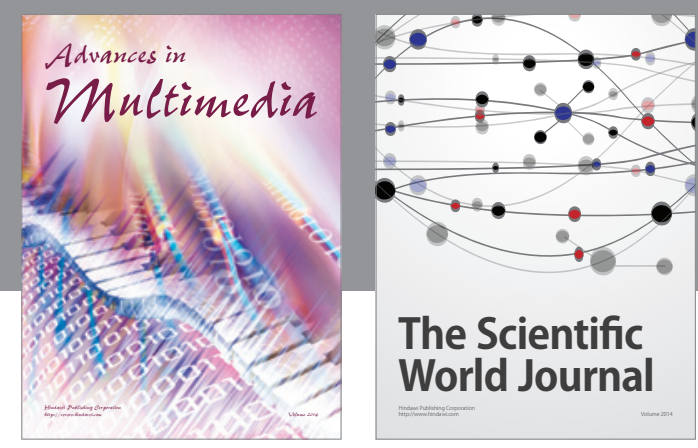

The Scientific World Journal
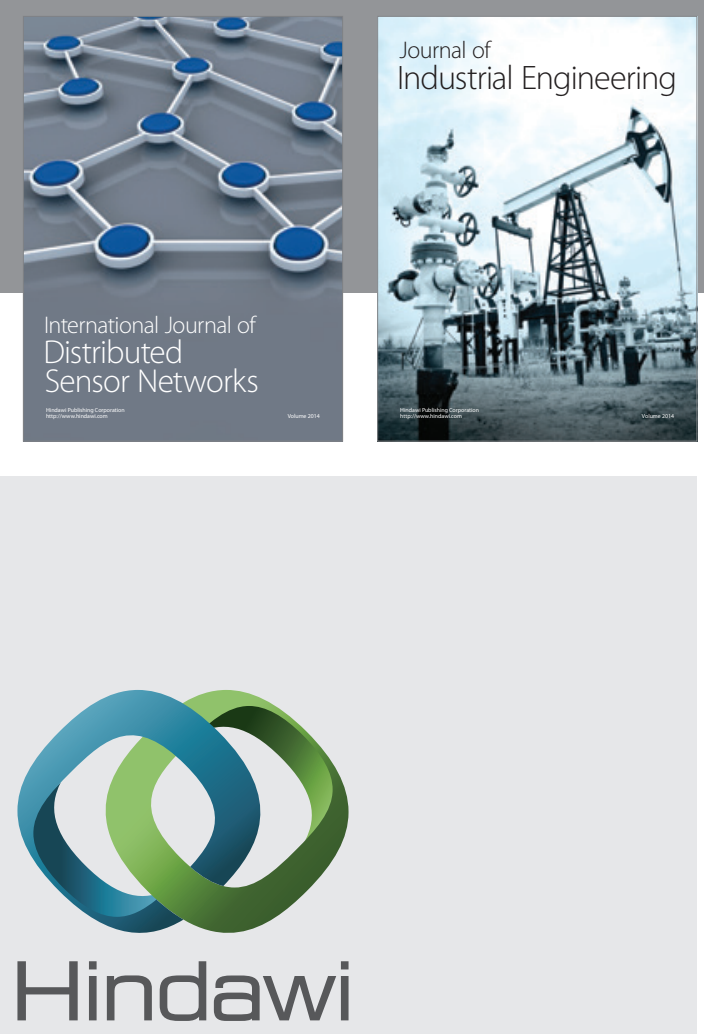

Submit your manuscripts at

http://www.hindawi.com

\section{Computer Networks} and Communications
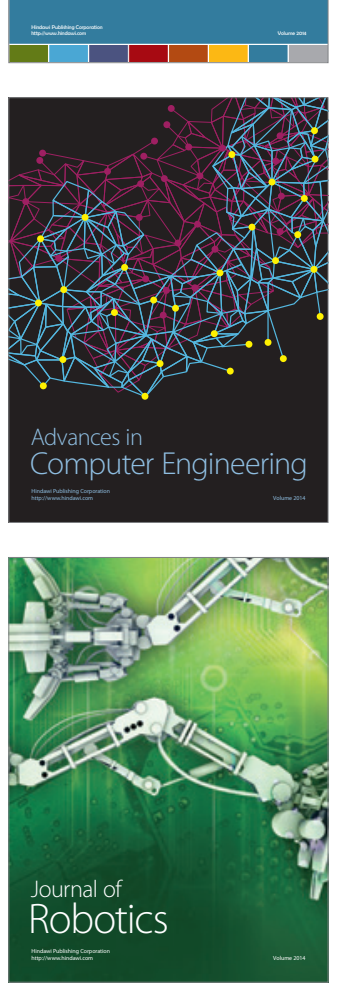
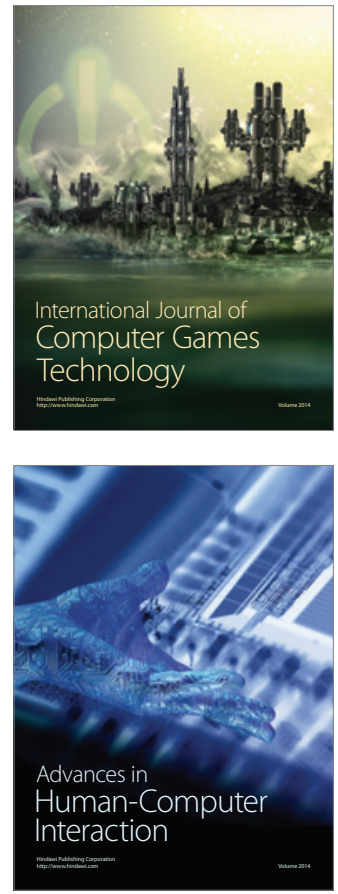
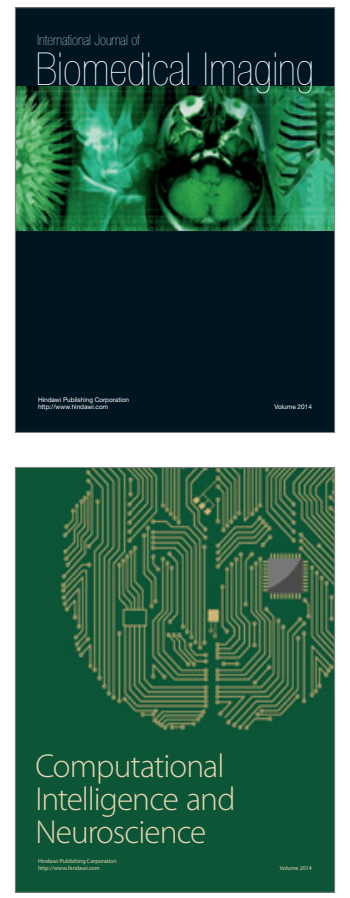
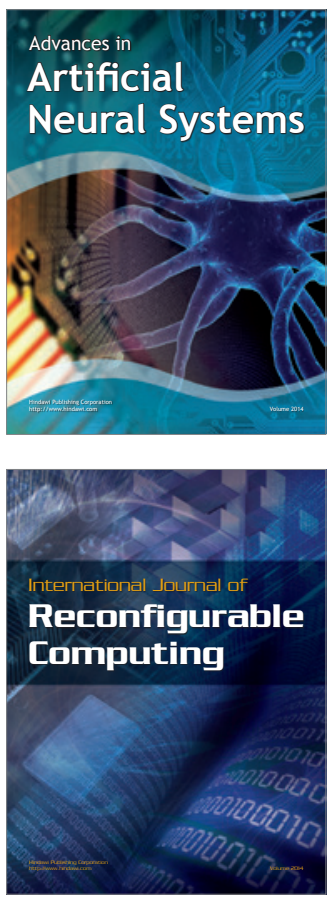
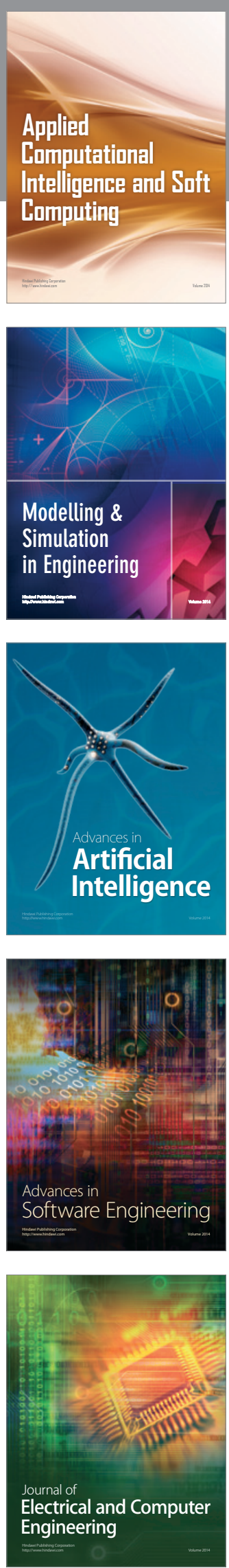